Dhaka Univ. J. Biol. Sci. 22(2): 109-117, 2013 (July)

\title{
IMPACT OF BIRTH COMPLICATION AND EARLY MEDICAL ILLNESS ON COGNITIVE ABILITIES AND ACADEMIC ATTAINMENT OF CHILDREN AND ADOLESCENTS
}

\author{
Mst. Afroza Parvin ${ }^{1}$ and Shaheen Islam* \\ Department of Educational \& Counselling Psychology, University of Dhaka, \\ Dhaka-1000, Bangladesh
}

Key words: Birth complication, Working memory, Verbal working memory

\begin{abstract}
The study focused on birth complication and early medical illness as detrimental developmental factors having adverse impact on children and adolescents' cognitive abilities and academic attainment. To assess cognitive abilities Wechsler Intelligence Scale for Children - Fourth edition (WISC-IV) was administered on 44 ( 26 boys, 18 girls) students of primary and secondary level schools of Dhaka city. The performance of last two consecutive school examinations was taken as a measure of academic attainment. The results revealed that higher percentages of students who had serious medical illness during early childhood demonstrated poor academic attainment. It has also revealed significant difference in students' working memory as well as verbal working memory in terms of academic achievements and serious medical illness during childhood. The results of this study have implications for early screening of children with developmental risk factors for early identification of possible poor cognitive abilities and poor academic attainment.
\end{abstract}

\section{Introduction}

Childhood is considered as a critical period of cognitive development and future academic achievement. Pre-maturation, birth complications, and serious medical illness during early developmental years have often been considered as risk factors for later cognitive growth and development.

Cognitive functioning of children is affected by environmental and health-related factors $^{(1-3)}$. Risk factors that interfere with cognitive functioning are especially important during early years of development because evidence showed that great majority of physical brain development occurs by the age of three years( ${ }^{(4)}$. Novello et al..$^{(4)}$ found that low birth weight, recurring illness, and chronic malnutrition lead to poor health, which in turn often leads to poor school achievement and early school leaving. It is also well documented that the early years of development is an essential base for later learning, behaviour and health ${ }^{(5)}$.

*Author for correspondence: <shaheen_islam@yahoo.com>. ${ }^{1}$ Department of Psychology, University of Dhaka, Dhaka-1000, Bangladesh. 
Impact of birth complication: Birth complication is a generic term which includes the following three indicators: (1) prenatal complications which consist of hypertension (measured by blood pressure above 140/90), low blood albumin level, bleeding, and other illness (diabetes, TB, pneumonia, asthma and epilepsy), (2) perinatal complications which consist of difficult foetal presentations (e.g., breech birth), premature rupture of the membrane, instrument delivery (forceps and caesarean), and foetal distress (difficulty in breathing) and (3) postnatal complications as indicated by the following two signs: cyanosis and treatment with oxygen.

Evidence has shown adverse impact of birth complications on child's cognitive growth and development. Sarah(6) noted that babies born naturally may have higher IQs than those delivered by caesarean section. According to scientists, when women give birth naturally there are higher levels of a special protein in babies' brains that helps boost intelligence levels as they develop. Scientists at Yale University in the US noted the increased levels of the protein, called UCP2, in babies born naturally could help foster their short and long term memories - key components of the human IQ - as they grow up $^{(6)}$.

Oxygen deprivation during birth process can result in serious injuries to newborn. While many newborns that suffer from insufficient oxygen during birth may not experience detectable brain damage they remain at a higher risk of learning disabilities, language delays and memory deficits ${ }^{(3)}$.

Impact of serious medical illness: Serious medical illness during early childhood is another developmental factor often treated as harmful for later development and growth. Guerrant et al.(7) noted that diarrhoea during infancy was negatively correlated with three tests of cognitive function in Brazilian children. Douglas et al.(8) noted that diarrhoea prevalence in the second year of life was significantly associated with cognitive test scores at the age between 12 and 24 months, a 1-day-increase in diarrhoea per month was associated with a decrease in WISC-R scores. In a study 46 children (age 6 - 10 years) with complete diarrhoea surveillance during their first 2 years of life were given a battery of five cognitive tests. Niehaus et al.(9) found that Test of Non-Verbal Intelligence-III (TONI) scores were inversely correlated with early childhood diarrhoea $(\mathrm{p}<0.01)$, even when controlling for maternal education, duration of breast-feeding, and early childhood helminthiasis (Ascaris or Trichuris). They also found that Wechsler Intelligence Scale for Children (WISC-III) Coding Tasks and WISC-III Digit Span (reverse and total) scores were also significantly lower in the 17 children with a history of early childhood persistent diarrhoea (PD; $\mathrm{p}<0.05)$, even when controlling for helminths and maternal education ${ }^{(9)}$.

As object permanence is considered the earliest method for assessing working memory, Loew et al. ${ }^{(10)}$ examined a sample of 233 children of 18 to 22 months old born with extremely low birth weight to identify factors affecting performance of object 
permanence. They found that children with lower birth weight and higher illness severity would have more difficulty on the object permanence task, and that no ethnic/racial differences would be found, as this is considered a culturally unbiased task.

The impact of pregnancy complications and prenatal threat on later cognitive growth and development of children were investigated in the context of Bangladesh. It was found that mothers who experienced greater threat like 'high blood pressure', 'bleeding', in the first trimester, 'infection' or other problem during prenatal period had 2.73 times more probability of having mentally retarded children than those without such threats ${ }^{(11)}$. Therefore, the impact birth complications and serious medical illness during early childhood on specific cognitive abilities and their link with later academic performance was therefore become a notion of this study. As working memory and verbal working memory are generally considered as good predictors of IQ and academic attainment, the present study focused on those two key factors of cognitive ability. The term 'working memory' is used to describe the ability we have to hold in mind and mentally manipulate information over short periods of time. Working memory is often thought of as a mental workspace that we can use to store important information in the course of our mental activities. And verbal working memory is a form of working memory that appears to play a significant role in language comprehension and problem-solving. According to Baddeley(12), verbal working memory has three components: (i) a buffer to store phonological codes, (ii) a rehearsal process to refresh those codes, and (iii) a set of executive processes to manipulate the contents of the buffer. Listening recall task, counting recall task, and digit span backward are some measures of verbal working memory.

The present study was undertaken to investigate the impact of serious medical illness during childhood and birth complication on working memory, verbal working memory and academic attainment.

\section{Materials and Methods}

Sample: The sample comprised of 44 school children between the age of 8 to 16 years selected from 4 government and non-government schools located in Dhaka city. Out of the total sample 26 were boys and 18 were girls. A random sample of 5 schools was drawn from a list of 15 government and non-government schools under the Board of Intermediate and Secondary Education following National Curriculum from three Thanas conveniently selected from south west zone of Dhaka city to cover wide range of socio-economic class. From these schools sample for two groups of students were selected in following ways.

Lists of students who had (1) failed in more than one subject (Lower attainment group), and (2) achieved in all subjects (Higher attainment group) in the last two consecutive examinations were prepared based on academic records maintained by the 
schools. An informed consent letter explaining the purpose of the study was circulated among them and those who gave consent took part in the study. Both the groups were matched in terms of sex, grade and schools. Sample with required criteria for lower attainment group was not available from one school and thus was excluded.

Measuring instruments: The Wechsler Intelligence Scale for Children - Fourth Edition (WISC-IV) is an individually administered clinical instrument for assessing the cognitive ability of children aged 6 years through 16 years 11 months was taken as a measure for working memory and verbal working memory ${ }^{(13)}$. The WISC-IV having ten core subtests are divided into four indices such as: verbal comprehension, perceptual reasoning, working memory and processing speed (Fig. 1).

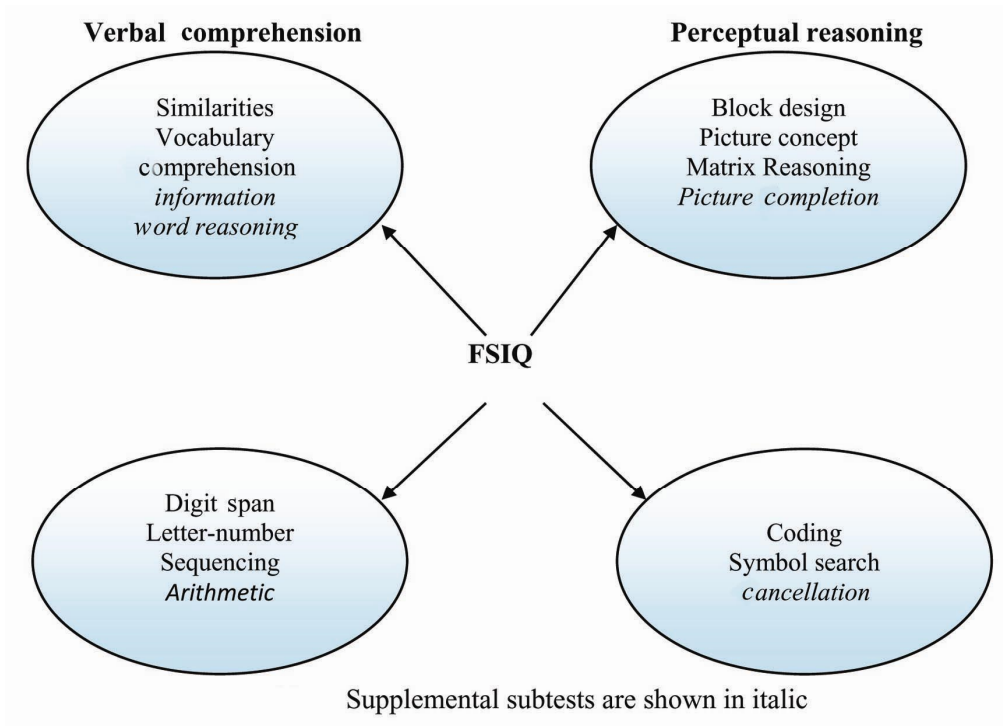

Fig. 1. WISC-IV Test Framework.

WISC-IV was used after translating the key instructions into Bangla. The test was administered on 30 students ( 15 boys and 15 girls) to determine psychometric properties of the Bangla translated instructions. Internal consistency, split-half reliability and testretest reliability were satisfactory. Alpha score of those subtests ranged from 0.71 to 0.92 . Split-half reliability was also measured for subtests (except coding, symbol search and cancellation) of WISC-IV and was found significant and satisfactory. For core subtests of WISC-IV, Guttman split-half reliability coefficient ranged from 0.67 to 0.84 . But for block design it was 0.45 and for comprehension it was 0.57 . For supplemental subtests of WISC-IV, Guttman split-half reliability coefficient ranged from 0.60 to 0.79 . 
Test-retest reliability was computed for coding, symbol search and cancellation based on the scores of 30 students between age 6 and 16 years. Test-retest reliability coefficient ranged between 0.74 and 0.81 .

Significantly moderate to high correlations between scaled score of subtest and full scale IQ score as well as between scaled score of subtest and composite score within the same index provided the evidence of convergent validity. Moderate positive correlations were found between scaled score of subtest and Full scale IQ score for block design ( $r=$ $0.60)$, coding $(r=0.66)$ and arithmetic $(r=0.60)$ and high positive correlations were found for rest of the subtests, which ranged from 0.74 to 0.87 .

Positive correlation was found between sum of scaled scores of subtests of each index and scaled score of subtest within the same index. For Verbal comprehension index, Perceptual reasoning index, Working memory index and Processing Speed index, ranges of correlations were from 0.76 to $0.85,0.68$ to $0.92,0.79$ to $0.89,0.79$ to 0.85 , respectively.

Content validity was also determined on the basis of experts' opinion. Corresponding mothers were also interviewed to collect information related to birth complications and early serious medical illness.

Procedure: Permission to conduct the study from the schools was taken explaining the nature and purpose of the study. Each school provided a free classroom for the purpose. This ensured standard administration of the scale. Cognitive ability of selected students was assessed by Wechsler Intelligence scale for Children - Fourth Edition (WISC-IV) using the translated Bangla instructions. All of the procedural directions stated in the administration and scoring manual of WISC-IV have been maintained while using the test. The information related to birth complications and serious medical illness was collected from parents of those students by using the personal demographic questionnaire. It is important to mention that scaled scores were used to eliminate probable effect of age on data.

\section{Results and Discussion}

The collected data were analyzed by using $t$ test and Chi-square, the results of which are shown in Table 1.

Table 1. Percentages in parentheses of students according to serious medical illness and birth complication.

\begin{tabular}{lcc}
\hline Developmental factors & Yes & No \\
\hline Serious medical illness & $17(38.6)$ & $27(61.4)$ \\
Birth complication & $7(15.9)$ & $37(81.1)$ \\
\hline
\end{tabular}


Table 1 shows that $38.6 \%$ of the sample had history of serious medical illness during the first five years of life and $15.9 \%$ had complications during birth. Table 2 shows the distribution of types of serious medical illness and birth complication.

Table 2. Distribution of types of serious medical illness and birth complication.

\begin{tabular}{llcc}
\hline Developmental factors & Types & Frequency & Percentage \\
\hline Serious medical & Typhoid & 7 & 15.9 \\
illness & Pneumonia & 4 & 9.1 \\
& Diarrheal disease & 3 & 6.8 \\
& High fever & 3 & 6.8 \\
Birth complication & Oxygen deprivation & 3 & 6.8 \\
& Longer labor pain & 2 & 4.5 \\
& Forceps delivery & 1 & 2.3 \\
& Caesarean & 1 & 2.3 \\
\hline
\end{tabular}

Table 3. Mean differences of cognitive factors among two groups of students according to serious medical illness.

\begin{tabular}{|c|c|c|c|c|c|c|c|}
\hline \multirow[t]{2}{*}{ Cognitive factors } & \multicolumn{3}{|c|}{ Had serious medical illness } & \multicolumn{3}{|c|}{ Had no serious medical illness } & \multirow[t]{2}{*}{$\mathrm{t}$ value } \\
\hline & $\mathrm{N}$ & Mean & $\mathrm{Sd}$ & $\mathrm{N}$ & Mean & $\mathrm{Sd}$ & \\
\hline Working memory & 17 & 11.82 & 4.12 & 27 & 16.48 & 8.58 & $-2.08^{*}$ \\
\hline $\begin{array}{l}\text { Verbal working } \\
\text { memory }\end{array}$ & 17 & 7.35 & 1.99 & 27 & 10.11 & 3.04 & $-3.30 * *$ \\
\hline
\end{tabular}

Table 4. Mean differences of cognitive factors among two groups of students according to birth complication.

\begin{tabular}{lccccccc}
\hline \multirow{2}{*}{$\begin{array}{l}\text { Cognitive } \\
\text { factors }\end{array}$} & \multicolumn{3}{c}{$\begin{array}{c}\text { Had history of birth } \\
\text { complication }\end{array}$} & \multicolumn{3}{c}{$\begin{array}{c}\text { Had no history of birth } \\
\text { complication }\end{array}$} & t value \\
\cline { 2 - 8 } & $\mathrm{N}$ & Mean & $\mathrm{Sd}$ & $\mathrm{N}$ & Mean & $\mathrm{Sd}$ & \\
\hline Working memory & 7 & 14.00 & 6.70 & 37 & 14.81 & 7.71 & -0.260 \\
Verbal working memory & 7 & 8.86 & 2.27 & 37 & 9.08 & 3.07 & -0.180 \\
\hline
\end{tabular}

The results presented in Tables 3 and 4 indicate that students who had history of serious medical illness significantly differ from those who did not have serious medical illness in terms of their ability of working memory $(t=-2.08, p<0.05)$ and verbal working memory $(\mathrm{t}=-3.03, \mathrm{p}<0.01)$. However, students who had history of birth complication did not differ significantly from those who did not have history of birth complication in terms of their ability of working memory and verbal working memory. 
Results of Table 5 show that large number (27.5\%) of lower attainment group had history of serious medical illness than higher attainment group. But no such difference was found for history of birth complication among the two attainment groups (Table 6).

The study partially highlighted the significance of adverse condition at early development age as having impact on low cognitive ability and academic attainment. Birth complication and serious medical illness during early childhood were considered as developmental factors in the present study. Working memory and verbal working memory were taken into consideration as cognitive ability.

Table 5. Relationship between serious medical illness and academic attainment.

\begin{tabular}{lccc}
\hline Serious medical illness & Lower attainment & Higher attainment & Total \\
\hline Yes & $12(27.5 \%)$ & $5(11.4 \%)$ & $17(38.6 \%)$ \\
No & $10(22.7 \%)$ & $17(38.6 \%)$ & $27(61.4 \%)$ \\
Total & $22(50 \%)$ & $22(50 \%)$ & $44(100 \%)$ \\
\hline
\end{tabular}

$\chi^{2}=4.69, \mathrm{p}<0.05$

Table 6. Relationship between birth complication and academic attainment.

\begin{tabular}{lccc}
\hline Birth complication & Lower attainment & Higher attainment & Total \\
\hline Yes & $4(9.1 \%)$ & $3(6.8 \%)$ & $7(15.9 \%)$ \\
No & $18(40.9 \%)$ & $19(43.2 \%)$ & $37(81.1 \%)$ \\
Total & $22(50 \%)$ & $22(50 \%)$ & $44(100 \%)$ \\
\hline
\end{tabular}

$\chi^{2}=0.170$.

Results of the study propagate that history of serious medical illness during the first five years of development may have an adverse impact on cognitive ability as depicted in lower score on working memory as well as verbal working memory (Table 3). Academic attainment was also found to be significantly poor (Table 5). Among the total number of students $(38.6 \%)$ with serious medical illness, $27.5 \%$ showed poor academic attainment. This finding is partly supported by Novello et al.(4) who found serious recurrent illness leading to poor academic attainment. This finding is also consistent with a longitudinal study conducted by Alloway and Alloway ${ }^{(14)}$ where child's working memory at 5 years was evident as a better predictor of academic success than IQ. Therefore, it may be assumed that serious medical illness at early age may result in poor cognitive ability (working memory and verbal working memory) which in turn might lead to poor academic attainment.

History of birth complications did not have similar effect either on cognitive ability or academic attainment (Tables 4 and 6). The finding was not consistent with the study of Sarah ${ }^{(4)}$ where it was reported that babies born naturally may have higher IQs than those delivered by caesarean section. This finding also seems contradictory with findings by 
Collins and Collins ${ }^{(3)}$. One of the possibilities of such disagreements may be due to lower number of birth complication $(15.9 \%)$ in the present study sample, retrospective nature of data or subsequent environmental factors that may reduce the effect of such early adversity. Cumulative stress of environmental, social and host factors may cause persistent disparity in pregnancy outcomes ${ }^{(15)}$. Studies have shown that sociodemographic factors have a greater total impact on adverse educational outcomes than early developmental factors ${ }^{(16)}$. Similarly, effect of environmental complexity on the apparent neurodevelopmental outcome of birth complications was manifested ${ }^{(17)}$.

Moreover, the sample was taken only from one zone of Dhaka city and the sample size was not large enough to reach any conclusive decision. Consideration of sociodemographic data and severity might have given a differential scenario. Differential effect of socio-environmental risk factors on benefits of intervention was suggested by Schatz et al.(18). Therefore, the study addresses the importance of both early developmental complexities as well as an enriched environment as contributing factor for cognitive ability and academic attainment. More controlled, longitudinal study incorporating possible environmental influences would be more contributing to the knowledge domain.

In spite of these limitations, the present study indicates a vast area of exploration and prediction for cognitive ability and academic attainment of children. It invites introduction of medical record, early screening procedure for early detection of children at risk who might benefit from early intervention with enriched environmental stimulation.

\section{References}

1. Bronfenbrenner U 1979. The ecology of human development: experiments by nature and by design. Cambridge: Harvard University Press.

2. Brown JL and E Pollitt 1996. Malnutrition, poverty and intellectual development. Scientific American 274: 38-43.

3. Collins and PC Collins 2012. Oxygen deprivation at birth: Cooling blankets becoming standard treatment.

4. Novello A C, C Degraw and D Kleinman 1992. Healthy children ready to learn: An essential collaboration between health and education. Public Health Report 107(1): 3-15.

5. McCain M N and JF Mustard 1999. The early years study: Reversing the real brain drain. Final Reports. Publications Ontario, Toronto.

6. Sarah J 2012. Babies born naturally 'have higher IQs than those delivered by caesarean section.

7. Guerrant D I, SR Moore, AA Lima, PD Patrick, JB Schorling, and RL Guerrant 1999. Association of early childhood diarrhoea and cryptosporidiosis with impaired physical fitness and cognitive function four-seven years later in a poor urban community in northeast Brazil. American Journal of Tropical Medicine and Hygiene 61(5): 707-713. 
8. Berkman DS, AG Lescano, RH Gilman, SL Lopez and MM Black 2002. Effects of stunting, diarrhoeal disease, and parasitic infection during infancy on cognition in late childhood: a follow-up study. The Lancet 359: 564-571.

9. Niehaus MD, SR Moore, PD Patrick, LL Derr, B Lorntz, AA Lima and RL Guerrant 2002. Early childhood diarrhea is associated with diminished cognitive function 4 to 7 years later in children in a northeast Brazilian Shantytown. American Journal of Tropical Medicine and Hygiene 66: 590-93.

10. Lowe J 2009. Early working memory in children born with extremely low birth weight: assessed by object permanence. Journal of Child Neurology 24(4): 410-5.

11. Islam S 2000. Pregnancy complication and prenatal threat associated with the risk of mental retardation, The Dhaka University Journal of Psychology 24: 1-8.

12. Baddeley A 2003. Working memory: looking back and looking forward. Nature Reviews. Neuroscience 4(10): 829-39.

13. Wechsler D 2003. WISC-IV technical and interpretive manual. San Antonio, TX: Psychological Corporation.

14. Alloway TP and RG Alloway 2010. Investigating the predictive roles of working memory and IQ in academic attainment. Journal of Experimental Child Psychology 80(2): 606-21.

15. Miranda ML, P Maxson and S Edwards 2009. Environmental contributions to disparities in Pregnancy Outcomes. Epidemiological Review 31(1): 67-83.

16. Resnick MB, RV Gueorguieva, RL Carter, M Ariet, Y Sun, J Roth and CS Mahan 1999. The impact of low birth weight, perinatal conditions, and socio-demographic factors on educational outcome in Kindergarten. Pediatrics 104(6): 74.

17. O'Brien F, S Roth, A Stewart, L Rifkin, T Rushe and J Wyatt 2004. The neuro-developmental progress of infants less than 33 weeks into adolescence. Archives of Disease in Childhood 89: 207-211.

18. Schatz J, R Finke and CW Roberts 2004. Interactions of biomedical and environmental risk factors for cognitive development: A preliminary study of sickle cell disease. Journal of Developmental \& Behavioral Pediatrics 25(5): 303-310. 